\title{
Fließgewässer und ihre Auen \\ Von der Siedlungsgeschichte zum vorbeugenden Hochwasser- und Biotopschutz in der Schweiz
}

\author{
Flußauen beherbergen eine hohe Artenvielfalt und dienen der Hochwasservorsorge. Sie gelten daher als \\ schützenswerte Landschaften. Der Schutzgedanke ist jedoch relativ neu. Über lange Jahre gingen in der \\ Schweiz Auengebiete ohne Ausgleich verloren. Flüsse wurden begradigt, Auengebiete \\ trockengelegt und in Ackerland umgewandelt, Kraftwerke und Kläranlagen wurden \\ an Flüssen gebaut und mußten gegen Überflutung gesichert werden. \\ Heute stehen noch verbliebene Auengebiete unter Schutz. Ein effektiver Biotop- und \\ Hochwasserschutz erfordert jedoch, daß Auenfläche in einem Umfang neu geschaffen \\ wird, der drei Prozent der landwirtschaftlichen Nutzfläche in der Schweiz entspricht.
}

Nino Kuhn

\begin{abstract}
$A^{\prime \prime}$ uen sind jene Bereiche von Flußtälern, welche der Fluß periodisch oder episodisch überflutet (Abbildung 1). Infolge komplexer geomorphologischer, klimatischer, hydrologischer und biologischer Prozesse sind Auen überaus dynamische Lebensräume (Ellenberg 1996, Reichholf 2005). In der Schweiz nannte man Auenwälder seit alters und vielerorts „Schachen“. Seit 1992 sind sie wegen ihrer hohen Biodiversität in der Schweiz Gegenstand der Verordnung über den Schutz der Auengebiete von nationaler Bedeutung (Auenverordnung), welche auf dem Biotopschutzartikel 18 des Bundesgesetzes vom 1. Juli 1966 über den Natur- und Heimatschutz (NHG) beruht. Dieser wurde allerdings erst 1987 beschlossen und 1988 rechtswirksam. Sowohl die Auenverordnung mit dem Aueninventar ${ }^{1}$ als auch die Fließgewässer mit ihren Auen waren geschichtlichen Entwicklungen unterworfen. Beiden Entwicklungen wird an dieser Stelle Rechnung getragen; vor allem werden die Maßnahmen vom Beginn des industriellen Zeitalters bis in die Gegenwart dargestellt, die zu massiven Landschaftsveränderungen in den Talebenen geführt haben. Die Auenverordnung steht heute am Anfang eines Prozesses, der einen effizienten Schutz von Auen zum Ziel hat. Ein solcher Schutz muß - über die noch vorhandenen naturnahen Reste hinaus revitalisierten und renaturierten Flußlandschaften gelten. In diese Richtung zielt auch der vorbeugende, nachhaltige Hochwasserschutz entsprechend dem neuen Bundesgesetz über den Wasserbau von 1991 und die Verordnung über den Wasserbau von 1999 (BWG 2001).

Der Schutz der Auen ist ein neues Phänomen in der Palette menschlicher Nutzung der Natur. Zwischen der Entwicklung des menschlichen Lebens in der Gemeinschaft und jener der Fließgewässer bestehen seit der Besiedlung des Landes enge Beziehungen. Die Bereitstellung von Nahrung (etwa durch Fischerei, Jagd
\end{abstract}

Kontakt: Dr. Nino Kuhn | Eidg. Forschungsanstalt für Wald, Schnee und Landschaft WSL | Zürcherstr. 111 | CH-8903 Birmensdorf | Tel.: +41/44/7392247 | Fax: +41/44/ 7392215 |

E-Mail: nino.kuhn@wsl.ch oder Ackerkulturen), die Energiegewinnung, der Gütertransport, die Wasserwirtschaft und die Abfallentsorgung (auch Entwässerung) waren schon immer unmittelbare praktische Nutzungsansprüche an Gewässer und Auen; Gewässerverschmutzung und Überschwemmungen eine Bedrohung von Leib und Gut. Mit den Ansprüchen und Risiken hat sich die Beziehung der Menschen zu Gewässern und dem diese begleitenden Pflanzenbestand gewandelt. Diesem Wandel sind die folgenden Skizzen gewidmet. Sie betonen einige Aspekte der Beeinflussung der Flußlandschaften, die bisher wenig Beachtung fanden oder anderer Fokussierung und Gewichtung unterlagen. Die geschichtliche Entwicklung der Fließgewässer ist in Anlehnung an Gerber (1967) dargestellt. Mit der Geschichte des Hochwasserschutzes in der Schweiz hat sich Vischer (2003) eingehend befaßt. Die Entwicklung im europäischen Rahmen faßte Hornsmann (1956) vortrefflich zusammen.

\section{Von der Natur- zur Kulturlandschaft in Flußtälern}

\section{Entwicklungsstufe: Leben mit der Natur - Mitgestaltung}

Der Mensch beschränkte sich lange Zeit auf Uferschutzmaßnahmen, in prähistorischer Zeit mit primitiven Mitteln, in der Römerzeit mit beachtlichem technischem Einsatz. Durch das ganze Mittelalter bis zum Beginn der technischen und industriellen Revolution wurde vorwiegend örtlich, unzusammenhängend und unkoordiniert gearbeitet. Die Flußlandschaften präsentierten sich weitgehend naturnah, jedenfalls was Artenreichtum und Zusammensetzung der Pflanzengemeinschaften anbelangt.

Vom Mittelalter bis in die Neuzeit wurden Flußanstößern von der Gemeinschaft die Wuhrpflicht und die Wasserwehr auferlegt. Die für den Uferschutz und den Bau strömungslenkender Buhnen, Holzkästen und Letzinen notwendigen Faschinen (Rutenbündel) entstammten dem Auenwald. So war dessen Nutzung für andere Zwecke eingeschränkt. In der Innerschweiz standen

1 Abrufbar unter www.auen.ch/index_de.html (abgerufen 03.05.2006). 
die Flußgehölze unter Bann, weil sie sonst für Köhlerei, Kalkbrand und andere Zwecke entfremdet worden wären (Sablonier 1990). Uferschutz wurde sowohl zum Schutz der Äcker im Überflutungsbereich als auch zur Erhaltung der Transportfähigkeit des Gewässers gefordert. Zu Zeiten, als weder Bahn noch Motorfahrzeuge existierten, waren Gewässer bevorzugte Transportwege. Davon zeugen Geschichtsschreibung und Sagen ebenso wie Redewendungen. So heißt es noch heute in Neuenburg und im Waadtland von einem Betrunkenen, er sei „nach Solothurn beladen“ (chargé pour Soleure): Tranken die Schiffsleute vom Wein, den sie auf der Zihl über Neuenburger See, Bieler See und Aare Richtung Solothurn transportierten, so blieben sie kaum nüchtern. Nicht nur Wein, Lebensmittel, Salz und andere Güter wurden auf Wasserwegen transportiert, sondern es wurde auch Holz getriftet und geflößt (Abbildung 2, S. 104).

Nach Grossmann (1972) galten seit 1365 folgende Flüsse als Floßstraßen: der Rhein mit seinen Zuflüssen in Graubünden, Vorarlberg und Baden-Württemberg, mit Thur, Töss, Wutach, Birs und Wiese, dann Aare mit Limmat, Reuss, Emme, Saane, Sense, Zihl und Broye, der Doubs, die Rhône mit Zuflüssen im Wallis, in den Leman und mit der Arve, der Tessin mit Brenno, Moësa, Verzasca und Maggia sowie der Inn. Für die Flößerei oder die Trift loser Stämme war genügend Wasser im Flußbett Voraussetzung. Wo dies nicht gegeben war, wurden Bäche und Flüsse gestaut. Dazu dienten aus Holzfachwerken gebaute Stauwehre oder Schleusen, auch Klausen genannt (Brändli und Dowhanytsch 2003).

Zur Transporttauglichkeit der Flüsse gehörte auch, daß die Flußufer auf „Treidelpfaden“ begehbar waren; denn Transportschiffe oder Boote mußten auch auf dem Rückweg den Wasserweg benutzen. Das Ziehen eines Schiffes mit Tier- oder Menschenkraft auf Flüssen und Kanälen vom Ufer aus nennt man „treideln“ (Treide = Zugtau). Nach Brockhaus wurden die ersten Treidelpfade in Mitteleuropa wahrscheinlich von den Merowingern und Karolingern angelegt und standen an den großen Strömen unter Königsschutz. Auch in der Schweiz sind sie historisch verbürgt. Zu Schiffereizwecken benutzt wurden Rhein, Aare, Limmat, Linth-Walensee, Rhône (Stadler 2004). Um Neuenburgerund Genfersee (Orbe-Venoge) zu verbinden, wurde 1640 der Bau des Canal d'Entreroches begonnen, 1648 jedoch wegen Finanzierungsschwierigkeiten abgebrochen. Er war Teil der Transhelvetischen Wasserstraße (SWV 1965). Die 16 Kilometer lange Strecke Yverdon-Entreroches wurde jedoch bis 1829 betrieben (Geographisches Lexikon der Schweiz 1904).

Aus den Überlieferungen ist zu folgern, daß Ströme und Flüsse mit ihren Auengebieten kaum je vom Menschen unbeeinflußt existierten. Solange aber die Ufersicherungen nicht tiefergreifende oder solidere Ausmaße annahmen, wurden die Fließgewässer bei episodischen Höchsthochwassern zurück zur Natur korrigiert.

\section{Entwicklungsstufe: Flußkorrektionen - Umgestaltung}

Um die zerstörerischen Überschwemmungen zu überwinden, wurden in der Schweiz im 18., besonders aber im 19. Jahrhundert massive flußbauliche Maßnahmen ein- und durchgeführt.

Die erste große Flußkorrektion war der Kanderdurchstich, also die Einleitung der Kander in den Thunersee 1711 bis 1714 . Dies veränderte das Abflußverhalten der Aare für alle unterliegenden Abschnitte grundlegend (Mühlberg 1885). Die Abflußmengen wurden massiv gedämpft, und das gesamte Geschiebe blieb im Thunersee. Ähnliche Wirkungen hatte für die Aare unterhalb des Bielersees der Bau des Hagneckkanals 1868 bis 1891 anläßlich der ersten Juragewässerkorrektion durch die Umleitung der Aare in den Bielersee. Auch die Korrektion der Emme hatte zur Folge, daß die Aare nicht mehr die Geschiebemengen für ein nachhaltiges Geschieberegime erhielt. Eine weitere Flußumlenkung in einem anderen Flußsystem galt der Linth in den Walensee bei der Linthkorrektion 1807 bis 1816.

Kanderdurchstich und Linthwerk waren neben der Birskorrektion die einschneidendsten Auenlandschaftsveränderungen vor 1850. Danach folgten Flußkorrektionen über das ganze Land verteilt. Bei allen außer dem Kanderdurchstich wurden gleichzeitig

ABBILDUNG 1: Auen sind die Bereiche von Flußtälern, welche der Fluß wiederholt überflutet. Die Auenvegetation - hier am Beispiel eines Flusses im Alpenvorland - ändert sich in Abhängigkeit von den WasserstandsKennwerten (nach Moor 1958).

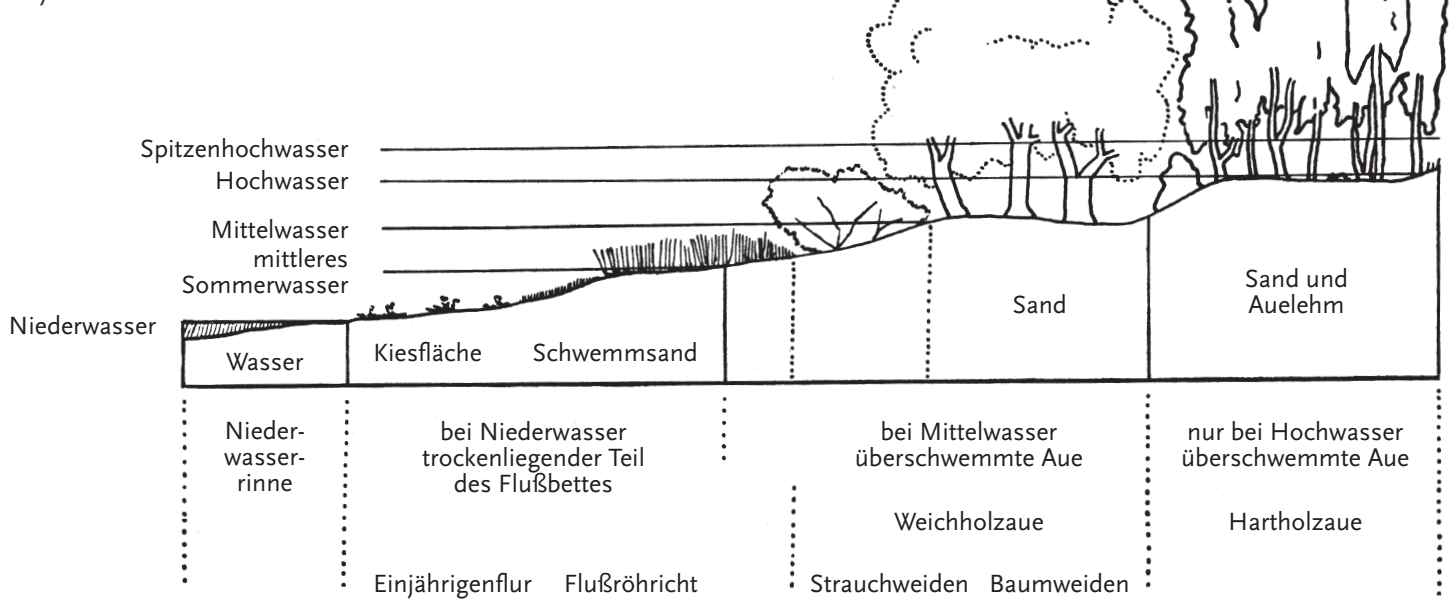


Hochwasserschutzdämme errichtet. Gegen 1920 waren die meisten umfassenden Flußkorrektionen abgeschlossen. Eine der letzten galt der Reusstalsanierung im Kanton Aargau zwischen 1971 und 1976. Allen Flußkorrektionen folgten umfangreiche Entwässerungen der ehemaligen Schwemmebenen (damals Meliorationen genannt, Glatthard 2003). Besonders wirksam wurden auch in der Schweiz Entwässerungen nach der Erfindung der Drainrohrpresse in England um 1840 (Weingarten und Kuratli 1993). Dadurch wurde eine umfassende landwirtschaftliche Nutzung der ursprünglichen Schwemmebenen eingeleitet und die Auen auf wenige kleine Reste reduziert.

ABBILDUNG 2: Gewässer waren lange Zeit wichtige Transportwege. Fang von 9000 Stämmen aus Holztrift in der Saane im Jahr 1894.

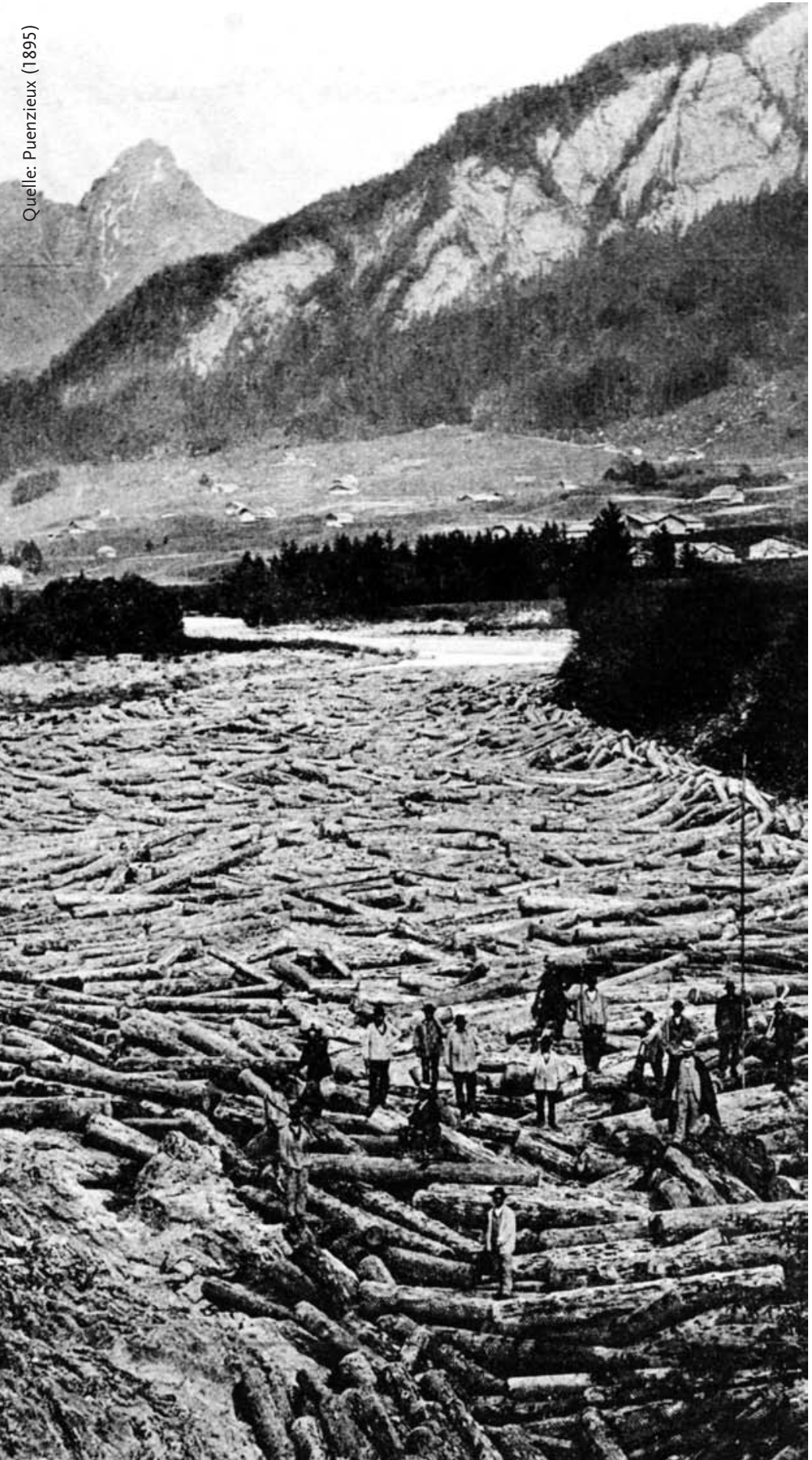

3. Entwicklungsstufe: Wasserkraftwerkbau - Umleitung, Kanalisierung, Befestigung

Auf die Flußkorrektionen folgten die Flußlaufkraftwerke, die den Auen den Raum direkt und nachhaltig streitig gemacht haben. Flußlaufkraftwerke verändern den Wasserhaushalt der Flußsysteme. Vielerorts wurden die Flußläufe einfach dadurch abgeschnitten, daß das Wasser in den Oberwasserkanal abgeleitet wurde (Ausleitungskraftwerke). Nur bei Hochwasser profitiert der ursprüngliche Flußlauf vom Wasserüberschuß und von Überflutung. Je perfekter die Abflußregelung und die Stauhaltung entlang des ganzen Flusses, desto seltener treten Hochwässer auf. Die Auenwälder werden in der floristischen Zusammensetzung und in den Bestandsstrukturen soweit verändert, daß lediglich kulissenartig angeordnete Baumreihen übrigbleiben. Deshalb fehlen die Voraussetzungen, sie als schutzwürdig zu bezeichnen.

Gemäß Daten des Bundesamtes für Wasser und Geologie wurde in der Schweiz bereits 1816 das erste Laufkraftwerk gebaut (Abbildung 3). Bei dieser in Oberuster errichteten Anlage muß es sich um eine Wasserkraftmaschine, also rein hydraulisch-mechanische Krafterzeugung gehandelt haben. Elektrische Energie konnte erst ab 1881 erzeugt werden, nachdem T. A. Edison 1879 einen zur Elektrizitätsversorgung tauglichen Generator gebaut hatte (Wolff 1993). Bereits am 1. Januar 1886 nahm die Spinnerei Huber in Uster ihren ersten Generator in Betrieb. Seither wurden in der Schweiz elektrizitätserzeugende Laufkraftwerke auf breiter Front und verteilt auf alle Flußgebiete errichtet. 2003 existierten 411 Laufkraftwerke mit über 300 Kilowatt Leistung. Laufkraftwerke wurden nicht kontinuierlich, sondern in Schüben in Betrieb genommen (Abbildung 3). Bächtold prognostizierte 1959: „Es ist bekannt, daß wir in 15 bis 20 Jahren mit dem Bau von Wasserkraftwerken am Ende sein werden, weil dann zumal alle wirtschaftlich nutzbaren Gewässer der Energiegewinnung dienstbar gemacht sein werden“ (Bächtold 1959). Um die Mitte der achtziger Jahre begann jedoch ein neue Welle: Zwischen 1986 und 2000 entstanden 52 neue Laufkraftwerke. Nur vier davon waren Dotierzentralen, also Anlagen, die Dotierwasser ${ }^{2}$ nutzen, bevor es zur Erfüllung ökologischer Zwecke in die Restwasserstrecke entlassen wird.

Die Speicherbecken von Speicherkraftwerken belegen mancherorts den Raum schützenswerter alpiner Schwemmlandschaften. Darüber hinaus beeinflussen sie das Abflußverhalten der Flüsse und damit die Gestalt der Auen. Dies weiter auszuführen, fehlt hier er Raum.

\section{Entwicklungsstufe: Gewässerschutz - gesundes Wasser}

Hygienisch unhaltbare Zustände mit Infektionskrankheiten und Epidemien durch verseuchtes Trinkwasser erforderten schon im ausgehenden 19. Jahrhundert Maßnahmen zum Schutz des Trinkwassers. St. Gallen nahm 1916 die erste Kläranlage in Be-

2 Dotierwasser muß vom bereits gefaßten Wasser in den Flußlauf zurückgegeben werden, wenn das Überlaufwasser das gesetzlich festgelegte Minimum der Restwassermenge nicht erreicht. 


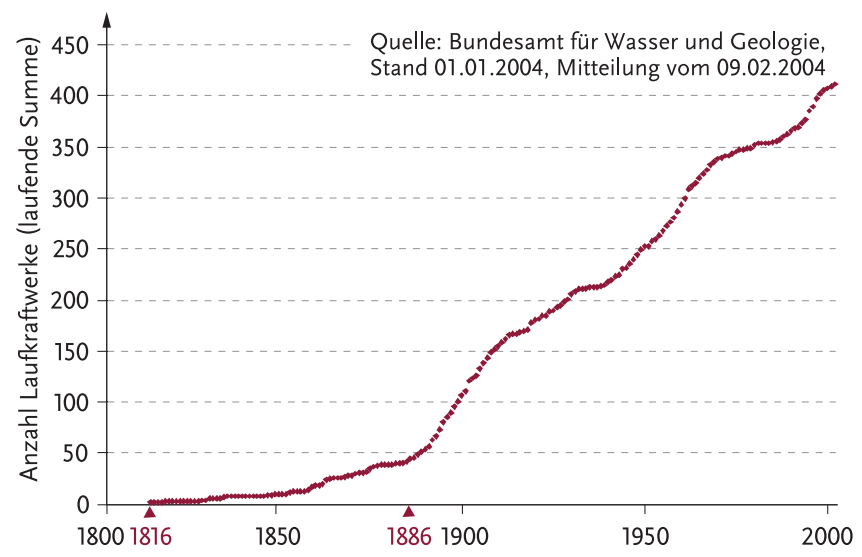

ABBILDUNG 3: Entwicklung des Laufkraftwerkbestandes in der Schweiz (laufende Summe der Anzahl von Laufkraftwerken $\geq 300 \mathrm{~kW}$ ). Das erste Laufkraftwerk wurde 1816 gebaut. Den ersten Generator zur Elektrizitätsversorgung nahm die Spinnerei Huber in Uster 1886 in Betrieb.

trieb. 1924 folgte die Stadt Zürich. Erst 1953 fand ein Gewässerschutzartikel Eingang in die Bundesverfassung. Das Gewässerschutzgesetz wurde 1957 in Kraft gesetzt, erlangte indessen erst Wirkung, als der Bund ab 1962 Beiträge an Abwasseranlagen ausrichtete (Zehnder 2000). Dadurch fand ein Übergang von der Wasserwirtschaft zur Wasserpflege statt, indem die Gewässerreinhaltung zur Trink- und Brauchwassergewinnung (reines, gesundes Wasser) Vorrang gewann. Die Wirkung der Kläranlagen auf die Auen muß differenziert betrachtet werden. In St. Gallen oder La Chaux-de-Fonds beanspruchen Kläranlagen den Flußraum nicht. Doch vielerorts wurden sie direkt in die Überflutungszone gebaut. Trotz unvollständiger Datenlage bleibt der Eindruck, daß viele Kläranlagen den Raum restlicher naturnaher Auengebiete beanspruchen. Wo dies der Fall ist, müssen Kläranlagen, wie auch Kraftwerke, vor Überschwemmung geschützt werden. Deshalb sind Auenschutz und Kläranlagen unverträgliche Nutzungen im Gewässerraum.

\section{Entwicklungsstufe: Natur- und Landschaftspflege - Nachhaltigkeitskultur im Hochwasserschutz}

Die Entwicklungsstufe der Natur- und Landschaftspflege begann um 1950. Während sich an den Alpenrand- und Mittellandseen schon früh Widerstand gegen die Überbauungen der Ufergestade regte (Verband zum Schutze des Landschaftsbildes am Zürichsee seit 1927, vergleiche Rebsamen et al. 2003; Verein Bielerseeschutz beziehungsweise Interessengemeinschaft Bielersee seit 1933; Verordnung zum Schutze des Greifensees im Kanton Zürich 1941 etc.), ließ man die Landschaftsveränderungen an den Fließgewässern geschehen. Auen galten eher als Ödland. Um bestehende Waldgebiete zu erhalten, wurde für die Rodung zugunsten der Landwirtschaft „Buschwald, speziell die Auenwaldungen an Aare, Emme, Reuss, Limmat, Töss, Thur, Rhône, Saane, Rhein, Tessin usw.“ empfohlen (Henne 1939). Erst als der Rheinfall und die Rheinau durch flußbauliche Maßnahmen zugunsten von Energiegewinnung und Hochrheinwasserstraße gefährdet schienen, formierte sich Widerstand. Erste Flugblätter (Am Rheinfall: Liebe
Jugend!) der Naturschutzkommission der Naturforschenden Gesellschaft Schaffhausen datieren vom Februar 1946. Das Überparteiliche Komitee zum Schutze der Stromlandschaft RheinfallRheinau bekämpfte zu Beginn der 1950er Jahre den Bau von Wasserkraftwerken in schützenswerten Fließgewässern. Dies tat auch das Komitee zum Schutze des Nationalparks wegen der Nutzung von Spöl und anderen Nationalparkgewässern, insbesondere auch wegen des Stausees, von dem befürchtet wurde, er werde das von Trockenheit geprägte inneralpine Klima weiträumig in ein Feuchtklima verwandeln. Beide Organisationen schlossen sich 1960 zum Rheinaubund - Schweizerische Arbeitsgemeinschaft für Natur und Heimat zusammen. Er wachte in der Folge mit Argusaugen über den Schutz der Fließgewässer. Allerdings ging es, wie bei den Seeufern, um den Schutz des Landschaftsbildes vor Überbauungen und weniger um Biotopschutz.

Die Initiative zum Schutze der Stromlandschaft RheinfallRheinau wurde am 5. Dezember 1954 verworfen. Doch die Aktion wurde zur Volksbewegung, die sich nicht mehr aufhalten ließ. Aus ihr heraus wurde noch 1954 der Keim der Kommission für die Inventarisation schweizerischer Landschaften und Naturdenkmäler von nationaler Bedeutung (KLN) gelegt (Schmassmann 1986). In dieser begannen 1959 die Schweizerische Vereinigung für Heimatschutz, der Schweizerische Bund für Naturschutz (SBN, heute Pro Natura) und der Schweizer Alpen-Club (SAC) gemeinsam eine Liste von Natur- und Kulturlandschaften aufzustellen, denen kantonsübergreifende nationale oder übernationale Bedeutung zukommt und die in ihrer charakteristischen Erscheinung absolut zu erhalten sind. Erklärtes Ziel der Verbände war es, von den Reaktionen auf kurz bevorstehende oder schon laufende Eingriffe in die Landschaft wegzukommen und statt dessen gemeinsam „A-priori-Schutzpostulate“ zu deklarieren. Bereits im Dezember 1955 wurde ein Fragebogen mit einem von den Präsidenten von Heimatschutz und SBN unterzeichneten Begleitschreiben an die kantonalen Naturschutzkommissionen, die Naturschutz-, Heimatschutz- sowie SAC-Sektionen und weitere Organisationen verschickt. Das erste KLN-Inventar wurde 1963 veröffentlicht; es enthielt 106 Objekte. Davon sind in 23 Objekten Flußlandschaften, Auenvegetation oder ähnliches von Bedeutung formuliert. In weiteren 13 Objekten wurde Wasserentnahme oder Kraftwerkbau als Bedrohung erwähnt, dem natürlichen Flußlauf also indirekt eine wesentliche Bedeutung in der Landschaft beigemessen. In mehr als einem Drittel aller Objekte spielten also bereits im ersten KLN-Inventar Flußlandschaften und Auenbiotope eine wichtige Rolle. Bis zur vierten und letzten Revision des KLN-Inventars, 1988, kamen weitere Flußlandschaften und Auenobjekte dazu. Das Bundesinventar der Landschaften und Naturdenkmäler von nationaler Bedeutung (BLN) hat die Postulate der KLN weitgehend übernommen (Kessler 1986). Es beruht auf Artikel 5 des Bundesgesetzes vom 1. Juli 1966 über den Natur- und Heimatschutz (NHG) und der Verordnung über das Bundesinventar der Landschaften und Naturdenkmäler (VBLN) vom 10. August 1977. Letztere hat sich in bezug auf den Auenschutz als Biotopschutz als zu wenig wirksam erwiesen, weshalb 1987 der Biotopschutzartikel 18 beschlossen wurde. 
Auch andernorts begann man, sich gegen Kraftwerke zu wehren, so etwa bei „der herrlichen Fluß- und Schachenlandschaft zwischen Brugg und Schinznach“ (Hauser 1950). Das Kraftwerk Wildegg-Brugg stand indessen 1949 bis 1952 im Bau.

Von Siegrist (1913) stammt eine unveröffentlichte Karte der damals noch intakten Flußauen in der Schweiz. Motivation seiner wissenschaftlichen Untersuchung der Auenwälder war allerdings das Phänomen des beständigen Wechsels der Vegetation im Erosionszyklus eines Flusses, welches die Auenlandschaft von den übrigen Landschaften abhebt. Von Schutz war mit keinem Wort die Rede. Noch 1952 dachte Siegrist nicht an Schutz oder Erhaltung von Auengebieten, sondern lediglich daran, die letzten Reste der „schönen, oft geradezu romantischen Gebilde der Auenwälder und die im Zusammenhang stehenden Pflanzengesellschaften in Wort und Bild der Nachwelt zu überliefern." So rief er in einem gedruckten Serienbrief in seiner Funktion als Aargauer Regierungs- und Nationalrat (und damit auch Verwaltungsrat der Elektrizitätswerke) zur Errichtung und jährlichen Speisung eines Fonds für die Erforschung der Pflanzengesellschaften schweizerischer Flußauen auf.

Die ersten wissenschaftlichen Arbeiten, die vom Auenfonds unterstützt wurden, waren jene von Moor (1958) und Müller (1958). Später konnten auch Heller $(1963,1969)$ und andere ihre Reiseauslagen vom Auenfonds begleichen lassen. Diese Arbeiten zählen zu den Grundlagen des Inventars der Auengebiete von nationaler Bedeutung.

\section{Ein neuer Blick auf Auengebiete}

\section{Mehr Raum den Fließgewässern}

Die Kampagne Mehr Raum den Fließgewässern leitet sich aus der Forderung nach einem effizienten Hochwasserschutz ab. Ausgedehnte Auengebiete können dazu einen wertvollen Beitrag leisten, denn sie sind als natürliche Rückhaltebecken in der Lage, Abflußspitzen zu vermindern. Demzufolge ist Auenschutz mit Hochwasserschutz gleichzusetzen.

Um so dramatischer ist der zu verzeichnende Rückgang der Auengebiete zu bewerten. Die Fläche der Auengebiete ist seit 1850 um 81076 Hektar zurückgegangen (siehe Tabelle). Dieser Rückgang entspricht 87 Prozent der ursprünglichen Ausdehnung der Fließgewässerauen (Müller-Wenk et al. 2003). ${ }^{3}$

In Anerkennung der Bedeutung der Auen für den Hochwasserschutz werden in jüngster Zeit Flüsse und Auen umfangreich revitalisiert. Auch die Gesetzgebung trägt dieser Bedeutung Rechnung: Im neuen Bundesgesetz vom 24. Januar 1991 über den Gewässerschutz (Gewässerschutzgesetz, GSchG) werden sehr viel mehr Ansprüche als bisher gewinnbringend koordiniert, so Wasserrechtsgesetz und Wasserbau, vor allem aber auch der Auenschutz (BWG 2001).

Die Reusstalsanierung im Kanton Aargau 1971 bis 1976 wurde noch gegen den starken Widerstand von Natur- und Landschaftsschutz durchgesetzt. Bundesgerichtlich geschützte Vergleiche trotzten dem Projekt indessen massive Konzessionen zum
TABELLE: Flächen der Fließgewässerauen in der Schweiz. Quellen: MüllerWenk et al. (2003), Aueninventar.

\begin{tabular}{|c|c|}
\hline Bezeichnung & läche in ha \\
\hline geomorphologische Schwemmebenen (ohne Seeflächen) & 290000 \\
\hline $\begin{array}{l}\text { Fläche der Fließgewässerauen um } 1850 \text { (historischer Referenz- } \\
\text { zeitpunkt; Mittelwert aus statistischer Erhebung auf Karten) }\end{array}$ & 129211 \\
\hline $\begin{array}{l}\text { Verlust der Fließgewässerauenfläche seit } 1850 \text { (Mittelwert aus } \\
\text { statistischer Erhebung auf Karten) }\end{array}$ & 81076 \\
\hline rezente Fließgewässerauenfläche (Arealstatistik) & 48135 \\
\hline $\begin{array}{l}\text { Bundesinventar der Auengebiete von nationaler Bedeutung } \\
\text { (Aueninventar) }\end{array}$ & 22618 \\
\hline - davon 216 Tieflagenauen & 13247 \\
\hline - davon 66 Gletschervorfelder und alpine Schwemmlandschaften & 9371 \\
\hline Zusatzbedarf an Auenschutzfläche & 32000 \\
\hline
\end{tabular}

Schutz der Flußlandschaft ab (Kessler und Maurer 1979). Sukzessive wurden etwa die Auenwälder von störenden Baumarten befreit, Hochwasserschutzdämme geöffnet oder versetzt, oder es wurde den Hochwässern neu zur Verfügung gestelltes Gelände modelliert. Kurz: An der Reuss wurde eine neue Ära des naturgerechten Hochwasserschutzes eingeleitet. Die Reusstalsanierung wurde zum Muster für umfassende Flußlandschafts-Revitalisierungen, etwa an Emme, Thur oder Rhône (Zaugg 2003).

Noch ist für einen wirksamen Hochwasserschutz viel Arbeit zu leisten. In der Urner Reussebene etwa mußte man einsehen, daß das Hochwasser von 1987 zwar unerwartet, aber doch vorhersehbar war (Zeller und Röthlisberger 1988, Fiebiger und Zollinger 1992, Petraschek 1992, Steinegger 1992). In dem engen Raum zwischen den Hochwasserdämmen konnten die außergewöhnlichen Wassermassen nicht rasch genug versickern. Schließlich zeigte sich auch bei den Starkniederschlägen im August 2005 mit enormen Zerstörungen durch Hangrutschungen, Murgänge und Hochwasser das gleiche Bild zu stark eingeengter Flußquerschnitte und zu risikoanfällig angesiedelter Ortschaften.

\section{Einflüsse der Agrar- und Arealpolitik sowie des Freizeitsports}

Alle früheren Flußkorrektionen, Entwässerungen und Meliorationen führten zu verbesserten Bedingungen für die landwirtschaftliche Produktion. Die Vergrößerung der Agrarfläche wurde mit dem Bedarf einer ständig steigenden Bevölkerungszahl begründet. Es verwundert nicht, daß am Gewässerrand ein Kampf gegen die Natur mit allen technischen Maßnahmen stattfand. Seit die Agrarpolitik gezwungen ist, sich am Weltmarkt zu orientieren, wird landwirtschaftliche Anbaufläche rückgebaut. Gefördert wird dies durch finanzielle Unterstützung des Bundes für extensiv genutzte Wiesen, Streuflächen, Grün-, Bunt- und Rotationsbrachen, Hecken-, Feld- und Ufergehölze oder für nachwachsende Rohstoffe (Faserpflanzen).

Die Agrarwirtschaft steckt indessen seit der ersten Agrarrevolution um 1850 in einem andauernden Strukturwandel, der nur zu Kriegs- und Krisenzeiten gebremst wurde (Baumann und Moser 2006). In neuerer Zeit ist die Öffnung der Märkte im Globalisierungstrend für den Paradigmenwechsel in der schweizerischen 
Agrarpolitik verantwortlich. Der Anspruch, die vollständige Versorgung der Bevölkerung zu leisten, ist längst Vergangenheit. Und die Importe von Landwirtschaftsprodukten bewirken laufend Preissenkungen, welche die schweizerischen Produzent(inn)en vor gewaltige Einkommens- und damit Existenzprobleme stellt.

Aus den Existenzproblemen, dem Generationenwechsel und dem Strukturwandel der landwirtschaftlichen Betriebe folgt, daß landwirtschaftliches Areal in nie dagewesenem Ausmaß verfügbar ist. Verschiedene Gesellschaftskreise haben verschiedene Nutzungsinteressen an diesen Flächen. Als Beispiel sei der Golfsport genannt, der derzeit als Ausdruck der modernen Fitneß-, Wellness-, Spiel- und Freizeitgesellschaft einen Boom erlebt. Zwischen 1990 und 2005 nahm die Fläche der Golfplätze in der Schweiz stetig zu: Von etwas mehr als 60 Neun-Loch-Einheiten auf mehr als 160 Neun-Loch-Einheiten. Diese rasante Zunahme seit 1990 ist aber auch ein Indikator für die Schwerpunkte in der Agrarpolitik, vor allem deren Arealpolitik. In der Schweiz gibt es heute 97 Golfplätze; von zwölfen ist die Flächenbelegung bekannt. Daraus läßt sich eine mittlere Fläche von 3,22 Hektar pro Loch berechnen. Dem Bestand von 1463 Löchern im Jahre 2005 entspricht eine Flächenbelegung von etwa 4710 Hektar in der gesamten Schweiz. Diese Fläche wiederum entspricht fast einem Drittel der nationalen Flächen für „Erholungs- und Grünanlagen“ (Statistik Schweiz, 2. Raum und Umwelt 1992/97: 15860 Hektar).

Die Anlage von Golfplätzen ist nicht nur eine raumwirksame Maßnahme im Sinne des Planungsrechts; sie unterliegt auch dem Baurecht. Deshalb bedürfen Golfplätze der öffentlichen Planauflage, bei der politische Auseinandersetzungen stattfinden. Aus diesem Grund hinkt die Anlage neuer oder die Erweiterung bestehender Golfplätze der Nachfrage hinterher. Derzeit sind über 60 Golf-Initialgruppen auf der Suche nach Plätzen. Daher ist in den nächsten zehn Jahren nicht mit einer Trendwende, sondern mit einer weiteren Ausweitung zu rechnen (Weber 2003).

\section{Vom Auenschutz zum Umbau der Flußlandschaften}

Zu den Nutznießern freiwerdenden Agrarareals sollte auch der Auenschutz gehören. Artikel 18 des Bundesgesetzes über den Natur- und Heimatschutz bestimmt grundsätzlich: „Dem Aussterben einheimischer Tier- und Pflanzenarten ist durch die Erhaltung genügend großer Lebensräume (Biotope) und andere geeignete Maßnahmen entgegenzuwirken. "Für die Artenvielfalt der Biotope spielt deren Ausdehnung eine entscheidende Rolle. Was die Flußauen anbelangt, ist festzustellen, daß deren Objekte von nationaler Bedeutung den Schutz der Auenverordnung genießen. Müller-Wenk et al. (2003) gehen davon aus, daß zu Beginn des industriellen Zeitalters um 1850 (Referenzzeitpunkt) Flora und Fauna im Biotopbereich der Fließgewässer noch nicht wesentlich beeinträchtigt waren. ${ }^{4}$ Dabei ist zu berücksichtigen,

3 Diese Zahl vergleicht die Landflächen der Auengebiete (ohne Gewässeranteile). 4 Der Zeitschnitt seit 1850 wurde gewählt, weil sich der Zustand der Flußlandschaften aus der flächendeckenden Siegfriedkarte im Maßstab 1:25000 oder 1:50000 ermitteln und mit der heutigen Landeskarte vergleichen läßt. daß die Flußumlenkungen an Aare und Linth bereits vor dem Referenzzeitpunkt die Biotop- und Habitatstrukturen weitreichend veränderten. Auch hatten ackerbauliche Nutzung und Beweidung schon vor dem Referenzzeitpunkt stattgefunden; vereinzelt sind solche Nutzungen noch heute in den Alpen, verbreitet noch in Flußsystemen Osteuropas, etwa in den Karpaten (Brändli und Dowhanytsch 2003) oder im Kaukasus zu finden. Schließlich muß auf den schon im 16. Jahrhundert geltenden Einfluß der Waldrodung und extensiven Beweidung in den Einzugsgebieten der Flüsse hingewiesen werden. Sie bewirkten bei Starkniederschlägen und Hochwässern starke Erosion, die vielerorts zu übermäßiger Ablagerung von Geschiebe und Auelehmen, zur Hebung der Flußsohle und damit zu Versumpfungen führte (Vischer 2003). Dadurch wurde allerdings die Fläche der Schwemm- und Auenlandschaften vergrößert; massiv reduziert wurde sie erst wieder mit den Flußkorrektionen und Entwässerungen.

In den seit 1958 bekannten Vegetationserhebungen in Auengebieten wurden insgesamt 1050 Gefäßpflanzenarten registriert. Davon gehören immerhin $153 \mathrm{zu}$ den von Auenbedingungen abhängigen und nach der Roten Liste gefährdeten Arten (Landolt 1991, IUCN 2001). Die Vegetationsaufnahmen beschreiben Pflanzengemeinschaften, die sich unter den stark veränderten Abflußund anderen Bedingungen seit Beginn des 20. Jahrhunderts entwickelt haben. Die ursprünglichen Gegebenheiten in Flußlandschaften, wie sie etwa Gerber (1967) oder Lüdi (1933) skizzierten, lassen darauf schließen, daß die Artenzahlen zum Referenzzeitpunkt weit größer waren. Zumindest fehlen den rezenten Pflanzengemeinschaften viele der früher häufigen Alpenschwemmlinge. Tatsächlich umfaßt die Liste der Gefäßpflanzenarten aller Objekte des revidierten Aueninventars (also unter Berücksichtigung der Gletschervorfelder und alpinen Schwemmlandschaften) knapp 1500 Arten. Auen beherbergen deshalb auf geringer Fläche bereits die Hälfte der Gefäßpflanzenarten des gesamten Landes. Nach Rust-Dubié et al. (2006) ist die faunistische Biodiversität aufgrund des kleinräumigen Zusammentreffens von amphibischen Biotopen und Steppenelementen sowie von Wäldern, Gebüschformationen und Grasfluren mindestens ebenso hoch.

Das Aueninventar weist derzeit eine Fläche von 22618 Hektar aus (siehe Tabelle). Davon gehören allerdings nur 13247 Hektar zu den Auen im bisher genannten Sinn. 9371 Hektar wurden durch Erweiterung um die sehr ähnliche Kategorie von Gletschervorfeldern und alpinen Schwemmlandschaften hinzugefügt (siehe Tabelle). Diese sind vorwiegend dazu bestimmt, den Biotopschutz im Gebiet der Speicherkraftwerke sicherzustellen. Doch schon das erste Aueninventar enthielt einige Objekte in diesen Lebensräumen. Die Studie von Müller-Wenk et al. (2003) bezieht sich ausschließlich auf tiefergelegene Auengebiete. Sie erachtet zwar die Rückkehr zur historischen, zum Referenzzeitpunkt bestimmten Ausdehnung von Fließgewässerauen als unrealistisch, weist jedoch nach, daß durch eine Erweiterung des Auenareals um 32000 Hektar der Schutzstatus für 1050 Pflanzenarten stark verbessert würde (siehe Tabelle). Dies gilt vor allem für die 153 auenspezifischen Gefäßpflanzenarten der Roten Liste. Die genannte Fläche entspricht etwa zwei Fünfteln der verschwundenen Au- 
enfläche. Damit läßt sich aber - gemäß der nicht-linearen, nach oben abflachenden Arrhenius-Kurve für die Areal-Artenzahl-Beziehung - das Überleben von über 60 Prozent oder knapp 95 der gefährdeten Pflanzenarten sichern. Es wird erwartet, daß Auen überwiegend auf freiwerdendem bisherigem Landwirtschaftsland wiederhergestellt werden. Rund drei Prozent der gegenwärtigen landwirtschaftlichen Produktionsfläche würden benötigt, was unter der heutigen, am Weltmarkt orientierten Agrarpolitik als verkraftbar erachtet wird. Es bedarf erheblicher Anstrengungen zum Ausgleich des Flächenbedarfs, damit die Auenverordnung ihre Aufgabe erfüllen kann. Rohde (2004) hat nachgewiesen, daß sich in beschränkten Aufweitungen kurzer Flußstrecken die häufigen Auenarten rasch einstellen und damit das Erscheinungsbild der Flußaue positiv beeinflussen. Um seltene Arten der Roten Liste wiederanzusiedeln, sind indessen solche Maßnahmen nicht hinreichend. So kommen auch Rust-Dubié et al. (2006) zum Schluß, daß der bisherige Auenschutz für die Erhaltung der gefährdeten Fauna langfristig nicht ausreicht. Seltene und bedrohte Arten benötigen für ihre Wiederansiedlung größere Freiräume. Solche Freiräume können in vernetzten Flußlandschaften geschaffen werden, in denen eine stärkere Abflußdynamik mit entsprechenden Geschiebeumlagerungen möglich ist.

\section{Auenschutz zur Erhaltung der Biodiversität und zum Hochwasserschutz}

Fließgewässer mit ihren Auen genießen wegen hoher Biodiversität besonderen Biotopschutz (Abbildung 4). Der Bestand an Auengebieten ist in der Schweiz seit 1850 auf nahezu ein Zehntel der Fläche geschrumpft. Entscheidende Faktoren bei den massiven Landschaftsveränderungen waren die Flußkorrektionen mit nachfolgenden Entwässerungen (Meliorationen), der anschließende Bau von Laufkraftwerken und vermutlich Maßnahmen im Gewäs-

ABBILDUNG 4: Landschilf-Weißerlenwald in einem Voralpental: Bei Hochwasser wurde infolge erhöhten Fließwiderstands Geröll abgelagert. Den extremen Lebensbedingungen widerstehen nur besonders angepaßte Pflanzen- und Tierarten.

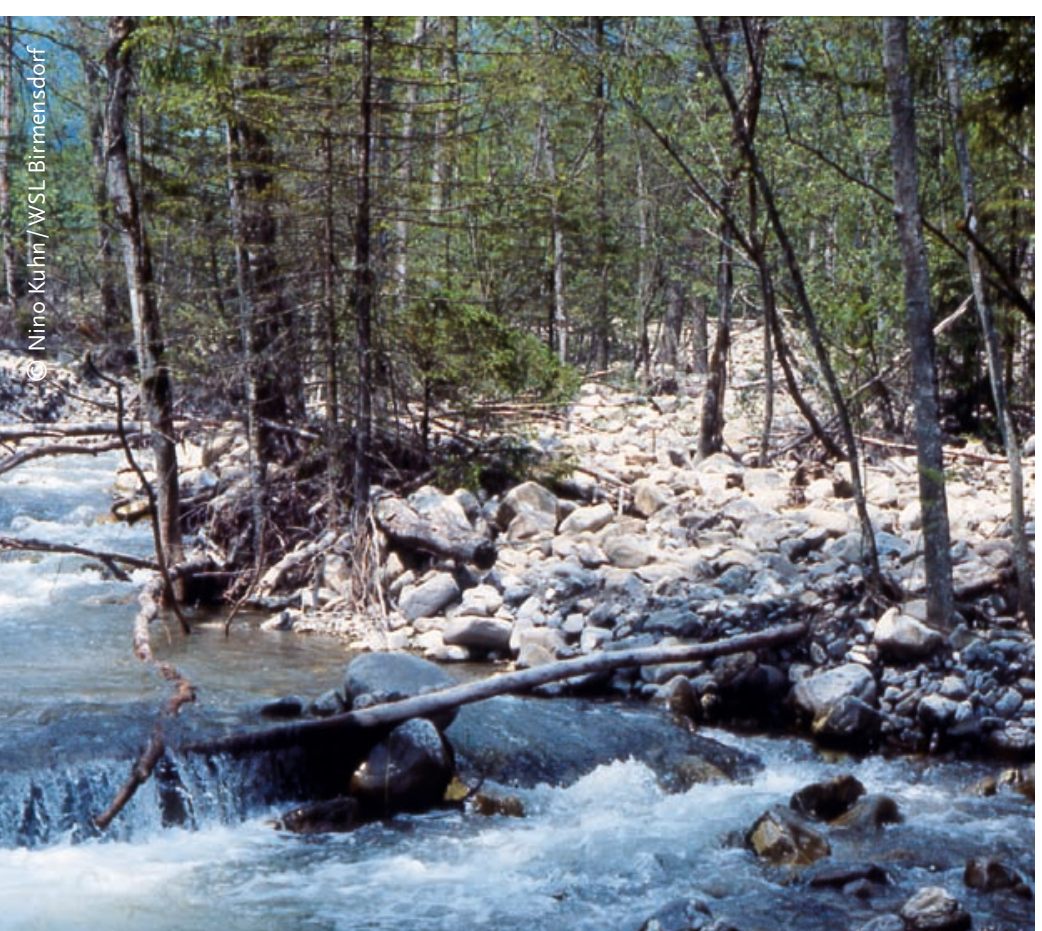

serschutz (Kläranlagen). Die heutige Entwicklung ist gekennzeichnet durch den Schutz bestehender Auengebiete (Auenverordnung), Revitalisierung von Flußlandschaften und nachhaltigen vorbeugenden Hochwasser- und Gewässerschutz unter Berücksichtigung von Arten-, Biotop-, Natur- und Landschaftsschutz. Damit die Auenverordnung ihr Ziel erreichen kann, nämlich das Aussterben einheimischer Tier- und Pflanzenarten in Flußlandschaft-Biotopen zu verhindern, ist Auenschutzfläche in der Größenordnung von 32000 Hektar neu zu schaffen. Angesichts der veränderten Bedingungen in der Agrarpolitik und der Forderungen des vorbeugenden Hochwasserschutzes nach Mehr Raum für die Fließgewässer scheint dies möglich.

Die Schweiz ist durch die Nutzung der Wasserkraft bei der Industrialisierung zu Wohlstand und Reichtum gelangt. Um die steigende Zahl von Einwohner(inne)n zu ernähren, wurden große Talebenen durch Flußkorrektionen und Entwässerungen der landwirtschaftlichen Produktion zugeführt. Dadurch sind Retentionsräume der Flüsse verlorengegangen; es kam zu Überflutungen. Die zum Schutz errichteten Hochwasserschutzdämme haben vielfach versagt.

Es ist daher unvermeidlich, daß wir heute zugunsten neuer Auenschutzfläche einen Teil der bisherigen landwirtschaftlichen Nutzflächen wieder aufgeben. Im Bereich der Flüsse sind sie in ein Hochwasserschutzkonzept einzubinden, das den Fließgewässern den Raum gewährt, den sie für einen wirksamen vorbeugenden Hochwasserschutz benötigen. Dadurch wird die Auenfläche vergrößert, und Flußrenaturierungen und -revitalisierungen werden ermöglicht. Für einen effizienten Flußlandschaftsschutz sind außer neuen Auenflächen naturnahe Abflußbedingungen unter Einschluß der Geschiebeführung sowie die Vernetzung der Flußlandschaftselemente unter sich sowie mit anderen Biotopen anzustreben. Der Umbau der Flußlandschaften in der Schweiz wird Jahrzehnte in Anspruch nehmen. Die notwendigen gesetzlichen Voraussetzungen sind heute sowohl von Seiten des Hochwasserals auch des Biotopschutzes gegeben. Sie sind konsequent zu beachten, anzuwenden und umzusetzen. Wichtig ist eine vorausschauende langfristige und weiträumige Planung, zum Beispiel bei den Konzessionserneuerungen für Flußkraftwerke, aber auch bei anderen baulichen und raumwirksamen Maßnahmen in Flußräumen. Dabei sind die bewährten Planungsverfahren der Raumplanung anzuwenden, die die Mehrfachinteressen an der Gewässernutzung berücksichtigen und die Konfliktpotentiale ermitteln (Landumlegungen, Nutzungsentflechtungen, Verlegung von Infrastruktureinrichtungen etc., siehe Schelbert 2005) und in öffentlichen Aushandlungsprozessen einer Lösung zuführen. Ohne Einbeziehung der privaten Landeigentümer (Abschluß von Bewirtschaftungsverträgen, Abgeltung von Ertragseinbußen etc.) ist dies nicht zu schaffen. Entscheidend ist auch das Verhalten der Elementarschaden-Versicherer. Auch bei ihnen hat eine neue Risikobeurteilung Einzug gehalten, indem sie etwa Wohn- und Gewerbebauten in Gefahrenzonen nicht mehr versichern.

Herzlichen Dank an die beiden anonymen Gutachter(innen), die mit ihren Anregungen das Manuskript verbessert haben. 


\section{Literatur}

Bächtold, J. 1959. Schweizerische Gesamtplanung im Kraftwerkbau. In: Natur und Technik in schweizerischer Schau und Verantwortung. Schriften der Neuen Helvetischen Gesellschaft. Herausgegeben von E. Egli. Atlantis Verlag.

Baumann, W., P. Moser. 2006. Landwirtschaft. Historisches Lexikon der Schweiz. Elektronische Publikation HLS, Version vom 01.03.2006.

Brändli, U.-B., J. Dowhanytsch. 2003. Urwälder im Zentrum Europas. Herausgegeben von Eidg. Forschungsanstalt WSL, Birmensdorf und KarpatenBiosphärenreservat, Rachiw. Bern: Haupt.

BWG (Bundesamt für Wasser und Geologie). 2001. Hochwasserschutz an Fliessgewässern, Wegleitung. Biel: Bundesamt für Wasser und Geologie.

Ellenberg, H. 1996. Vegetation Mitteleuropas mit den Alpen in ökologischer, dynamischer und historischer Sicht. 5. Auflage. Stuttgart: Ulmer.

Fiebiger, G., F. Zollinger. 1992. Schutz des Lebensraumes vor Hochwasser, Muren und Lawinen. Internationales Symposium Interpraevent 1992, Band 6, Schlussband. Bern.

Geographisches Lexikon der Schweiz. 1904. Entreroche. Zweiter Band. Neuenburg: Verlag von Gebrüder Attinger.

Gerber, E. 1967. Die Flußauen in der schweizerischen Kulturlandschaft. Geographica Helvetica 22: 1-26.

Glatthard, T. 2003. Melioration. Historisches Lexikon der Schweiz. Elektronische Publikation HLS, Version vom 01.09.2003.

Grossmann, H. 1972. Flösserei und Holzhandel aus den Schweizer Bergen bis zum Ende des 19. Jahrhunderts. Mitteilungen der Antiquarischen Gesellschaft Zürich 46/1: 92.

Hauser, W. R. 1950. Auenwälder - Dreistromland - Zum Geleit. Brugger Neujahrs-Blätter 60: 3.

Heller, H. 1963. Struktur und Dynamik von Auenwäldern. Beiträge zur Geobotanischen Landesaufnahme der Schweiz 42: 75.

Heller, H. 1969. Lebensbedingungen und Abfolge der Flussauenvegetation in der Schweiz. Mitteilungen der Schweizerischen Anstalt für das forstliche Versuchswesen 45/1: 1-145.

Henne, A. 1939. Einfluss des Schweizerischen Forstvereins auf die Entwicklung des Forstwesens in der Schweiz 1843-1938. Bern: Buchdruckerei Büchler \& Co.

Hornsmann, E. 1956. Wasser - Ein Problem jeder Zeit. Bern: Francke.

IUCN (The International Union for the Conservation of Nature and Natural Resources). 2001. Red List Categories and Criteria. Version 3.1.1. IUCN Species Survival Commission. Gland, CH, Cambridge, UK: IUCN.

Kessler, E. 1986. Erfahrung mit dem in der Schweiz im Aufbau begriffenen „Bundesinventar der Landschaften und Naturdenkmäler von nationaler Bedeutung". Schriftenreihe des deutschen Rates für Landespflege 50: 904-910.

Kessler, E., R. Maurer. 1979. Fallbeispiel Reusstalsanierung. Exkursion vom 20. September 1979. Herausgegeben vom Regierungsrat des Kantons Aargau für die 3. europäische Ministerkonferenz für Umweltschutz in der Schweiz (Verträglichkeit von Land- und Waldwirtschaft mit dem Umweltschutz). Aarau.

Landolt, E. 1991. Gefährdung der Farn- und Blütenpflanzen in der Schweiz. Bern: Bundesamt für Umwelt, Wald und Landschaft.

Lüdi, W. 1933. Das Grosse Moos im westschweizerischen Seelande und die Geschichte seiner Entstehung. Veröffentlichungen des Geobotanischen Institutes Rübel in Zürich 11.

Moor, M. 1958. Pflanzengesellschaften schweizerischer Flussauen. Mitteilungen der Schweizerischen Anstalt für das forstliche Versuchswesen 34/4: 221-360.

Mühlberg, F. 1885. Die heutigen und früheren Verhältnisse der Aare bei Aarau. Programm der Aargauischen Kantonsschule. Aarau.

Müller, M. 1958. Auenwaldböden des schweizerischen Mittellandes. Mitteilungen der Schweizerischen Anstalt für das forstliche Versuchswesen 34/2: 37-86.

Müller-Wenk, R., F. Huber, N. Kuhn, A. Peter. 2003. Landnutzung in potentiellen Fliessgewässer-Auen - Artengefährdung und Ökobilanzen. Schriftenreihe Umwelt Nr. 361. Bern: Bundesamt für Umwelt, Wald und Landschaft.

Petraschek, A. 1992. Die Ursachenanalyse der Hochwasser 1987. In: Schutz des Lebensraumes vor Hochwasser, Muren und Lawinen. Herausgegeben von G. Fiebiger, F. Zollinger. Internationales Symposium Interpraevent 1992, Band 6, Schlussband. Bern. 41-52.

Puenzieux, A. 1895. Le flottage des bois dans le canton de Vaud. Schweizerische Zeitschrift für Forstwesen Jahrgang 1895: 206-213.
Rebsamen, H.P., E. Angstmann, Z. Rarici-Ciprys. 2003. Zürichsee Landschaftsschutz 1927-2002. Stäfa, CH: Th. Gut Verlag.

Reichholf, J.H. 2005. Die Zukunft der Arten. Neue ökologische Überraschungen. München: C. H. Beck.

Rohde, S. 2004. River restoration: Potential and limitations to re-establish riparian landscapes. Assessment and planning. Dissertation, ETH Zürich.

Rust-Dubié, C., K. Schneider, T. Walter. 2006. Fauna der Schweizer Auen. Eine Datenbank für Praxis und Wissenschaft. Bristol-Schriftenreihe Band 16. Bern: Haupt.

Sablonier, R. 1990. Innerschweizer Gesellschaft im 14. Jahrhundert Sozialstruktur und Wirtschaft. In: Innerschweiz und frühe Eidgenossenschaft. Band 2. Herausgegeben von H. J. B. Achermann, J. Brülisauer, P. Hoppe. Olten: Walter-Verlag. 11-233.

Schelbert, B. 2005. Frösche würden Aargau wählen. Das Programm „Auenschutzpark Aargau“. Ornis 5/05: 4-8.

Schmassmann, H.J. 1986. Entstehung und Kriterien des schweizerischen Inventars der zu erhaltenden Landschaften und Naturdenkmäler von nationaler Bedeutung. Schriftenreihe des deutschen Rates für Landespflege 50: 901-903.

Siegrist, R. 1913. Die Auenwälder der Aare mit besonderer Beücksichtigung ihres genetischen Zusammenhanges mit anderen flussbegleitenden Pflanzengesellschaften. Dissertation, ETH Zürich.

Stadler, H. 2004. Flüsse. Historisches Lexikon der Schweiz. Elektronische Publikation HLS, Version vom 09.03.2004.

Steinegger, F. 1992. Leben in gefährdetem Raum - am Beispiel des Kantons Uri. In: Schutz des Lebensraumes vor Hochwasser, Muren und Lawinen. Herausgegeben von G. Fiebiger, F. Zollinger. Internationales Symposium Interpraevent 1992, Band 6, Schlussband. Bern. 25-32.

SWV (Schweizerischer Wasserwirtschaftsverband). 1965. Binnenschiffahrt und Gewässerschutz. Wasser- und Energiewirtschaft. Sonderheft. S.-K. f. B. u. Gewässerschutz. Baden: Schweizerischer Wasserwirtschaftsverband.

Vischer, D. 2003. Die Geschichte des Hochwasserschutzes in der Schweiz. Bern: Bundesamt für Wasser und Geologie.

Weber, D. 2003. Trends bei der Nachfrage nach Erholungsraum - Beispiel Golf. In: Heute Agrarpolitik - morgen eine integrale Grünraumpolitik für die Land(wirt)schaft in Agglomerationsräumen. Herausgegeben von A. Möhring, M. Pezzati. Agrarwirtschaft und Agrarsoziologie 2/03. Schweizerische Gesellschaft für Agrarwirtschaft und Agrarsoziologie.

Weingarten, E. K., B. Kuratli. 1993. Meliorationen. Schweizer Lexikon in sechs Bänden. Band 4. Luzern: Verlag Schweizer Lexikon Mengis und Ziehr.

Wolff, K. 1993. Kraftwerke. Schweizer Lexikon in sechs Bänden. Band 3. Luzern: Verlag Schweizer Lexikon Mengis und Ziehr.

Zaugg, M. 2003. Mehr Raum den Fließgewässern. Der Weg zu einem nachhaltigen Hochwasserschutz. GAIA 12/3: 201-207.

Zehnder, A. J. B. 2000. Gewässerschutz im Wasserschloss Europas. Neue Zürcher Zeitung, 18.05.2000.

Zeller, J., G. Röthlisberger. 1988. Unwetterschäden in der Schweiz im Jahre 1987. wasser, energie, luft 80/1-2: 29-42.

Nino Kuhn

Geboren 1936 in St. Gallen. 1958 bis 1962 Studium der

Forstwissenschaften an der ETH Zürich. 1962 bis 1966 Assistent am Geobotanischen Institut, Stiftung Rübel an der ETH Zürich. Dissertation bei Prof. Dr. Heinz Ellenberg. 1966 bis 2001 wissenschaftlicher Mitarbeiter für

Vegetationsökologie an der WSL. 1963 bis 1988 Sekretär der KLN (Kommission für die Inventarisation schweizerischer Landschaften und Naturdenkmäler von nationaler Bedeutung). 1980 bis 1988 Ausarbeitung des ersten Aueninventars der Schweiz im Auftrag des BUWAL (Bundesamt für Umwelt, Wald und Landschaft). 